Results Both the TLR4 antagonist's (STM28 and IAXO compound) reduced the plasma liver enzymes, ammonia and creatinine to the control level. The increase in the plasma TNF- $\alpha$ induced by APAP $(45 \pm 3.2)$ was attenuated following TLR4 antagonist (20 \pm 2.3$)$ $(p<0.01)$. This was associated with a reduction in brain water $(p<0.01)$. Both the TLR4 antagonists significantly reduced pericentral necrosis of the liver. Both these interventions showed an improvement in the survival as using the log rank test $(p<0.02)$. TLR4 KO mice treated with APAP were protected from liver necrosis and had significantly better survival than wild type controls $(\mathrm{p}<0.002)$.

Conclusion These data provides evidence for an important of TLR4 in APAP induced ALF and provide the rationale for a clinical trial of this strategy in ALF.

Competing interests None declared.

\section{OC-067 THE BALANCE BETWEEN T HELPER 17 AND F0XP3+ T REGULATORY CELLS IN PATIENTS WITH CHRONIC HEPATITIS C: RELATION TO DISEASE ACTIVITY AND HEPATIC FIBROSIS}

doi:10.1136/gutjnl-2012-302514a.67

${ }^{1} \mathrm{H}$ A El Aggan, ${ }^{*} \mathrm{~N}$ Farahat, ${ }^{3} \mathrm{~L}$ Younis, ${ }^{1} \mathrm{~A}$ ElYamany, ${ }^{1} \mathrm{D}$ Mostafa. ${ }^{1}$ Department of Medicine, Hepatobilary Unit; ${ }^{2}$ Department of Clinical Pathology; ${ }^{3}$ Department of Pathology, Faculty of medicine, Alexandria, Egypt

Introduction $\mathrm{T}$ helper (Th)17 cells, a newly identified subset of Th cells, are major mediators of inflammation-associated disease and have a reciprocal developmental relationship with the immunosuppressive $\mathrm{T}$ regulatory (Treg) cells, which actively restrain the inflammatory response. The present work was designed to study the balance between Th17 cells and Treg cells in patients with chronic hepatitis $\mathrm{C}(\mathrm{CHC})$ in relation to disease activity and severity of hepatic fibrosis.

Methods Twenty patients with treatment-naive $\mathrm{CHC}$ and 20 healthy subjects were included in the study. The Th cells, Th17 cells and Treg cell subsets in fresh whole blood samples were identified as $\mathrm{CD}^{+} \mathrm{CD}^{+}, \mathrm{CD}^{+} \mathrm{IL}_{17 \mathrm{~A}^{+}}$and $\mathrm{CD} 4^{+} \mathrm{CD} 25^{+} \mathrm{FoxP}^{+}$cells respectively using flow cytometry and expressed as percentages of total lymphocytic count. Serum IL17 levels were measured using solid phase sandwich enzyme linked immunosorbant assay kit. Liver biopsies from patients with CHC were examined to assess histological activity grade and fibrosis stage according to METAVIR scoring system. Liver-infiltrating $\mathrm{CD}^{+}$(Th cells), IL17A ${ }^{+}$cells (Th17 cells) and FoxP3 ${ }^{+}$cells (Treg cells) were detected by immunohistochemical staining and their proportions were determined as ratios of infiltrating $\mathrm{CD}^{+}$Th cells.

Results Patients with CHC showed significant increases in the percentage of Th17 cells, Th17 cells/FoxP3 ${ }^{+}$Treg cells ratio in peripheral blood and serum IL17 levels and a significant decrease in the percentage of circulating FoxP3 ${ }^{+}$Treg cells compared with healthy subjects $(p<0.01)$. The percentages of peripheral blood $\mathrm{CD}^{+}$Th cells were not statistically different between the two groups $(p=0.284)$. The proportions of liver-infiltrating IL17A ${ }^{+}$cells and $\mathrm{FoxP}^{+}$cells of the total intrahepatic $\mathrm{CD}^{+}{ }^{+}$cell population were inversely correlated and showed positive correlations with the percentages of circulating Th17 cells and FoxP3 ${ }^{+}$Treg cells respectively in patients with CHC $(p<0.05)$. The METAVIR necroinflammation grade and fibrosis stage [but not serum HCV RNA levels] were directly correlated with the proportion of intrahepatic IL17A $^{+}$cells and IL17A ${ }^{+}$cells/FoxP3 ${ }^{+}$cells ratio and serum IL17 levels and were inversely correlated with the proportion of liverinfiltrating FoxP3 ${ }^{+}$cells $(\mathrm{p}<0.05)$.

Conclusion In patients with $\mathrm{CHC}$, the $\mathrm{CD} 4^{+}$Th cell phenotype is skewed towards the IL17 producing-Th phenotype. The imbalance between Th17 and Foxp3 ${ }^{+}$Treg cells plays an important role in disease progression and hepatic fibrosis in $\mathrm{CHC}$.

Competing interests None declared.

\section{OC-068 ALBUMIN IS CENTRAL IN MEDIATING THE CARDIO- RENAL DYSFUNCTION OF CIRRHOSIS: A STUDY IN ANALBUMINAEMIC RATS}

doi:10.1136/gutjnl-2012-302514a.68

R Garcia Martinez, ${ }^{*}$ A Habtesion, G Tritto, M Jover, R Jalan, N Davies. Department of Medicine, UCL Hepatology, London, UK

Introduction Albumin is a multifunctional protein which is reduced in concentration and function in liver disease. Albumin infusion prevents and improves renal dysfunction in patients with advanced liver failure but the mechanisms of its beneficial properties are unclear. We hypothesised that albumin is central in the maintenance of cardio-renal function in cirrhosis and albumin impairment worsens outcome. In order to answer this question we investigated analbuminaemic rats, characterised by lack of albumin but with normal protein concentration, following induction of cirrhosis with bile duct ligation (BDL).

Methods Male Sprague-Dawley (SD) and Nagase analbuminaemic (NAR) rats were studied 6 weeks after BDL or sham surgery $(n=6$ sham-SD, 8 sham-NAR, 7 SD-BDL, 7 NAR-BDL). Rats underwent systemic mean arterial pressure (MAP) and portal pressure (PP) assessment under terminal anaesthesia. Plasma and urine were collected for measurements of renal function and protein profile. Plasma renin activity (PRA) was measured as a marker of cardiorenal dysfunction. Urinary neopterin, a marker of macrophage activation was assessed.

Results NARs showed plasma total protein concentration similar to SD despite lack of albumin before $(72 \pm 15$ vs $82 \pm 8)$ and after BDL (67 \pm 7 vs $75 \pm 22$ ). After BDL both groups of animals showed histological evidence of severe liver damage, though the NARs showed a significantly worse systemic haemodynamics with lower MAP ( $p=0.01)$, evidence of renal dysfunction indicated by higher plasma creatinine and higher PRA $(\mathrm{p}<0.05)$ compared with SD (Abstract OC-068 figure 1). There was a significant inflammatory response following BDL in both groups showed by an increase in urinary neopterin which was found to correlate with PRA ( $r=0.59$, $\mathrm{p}<0.01)$

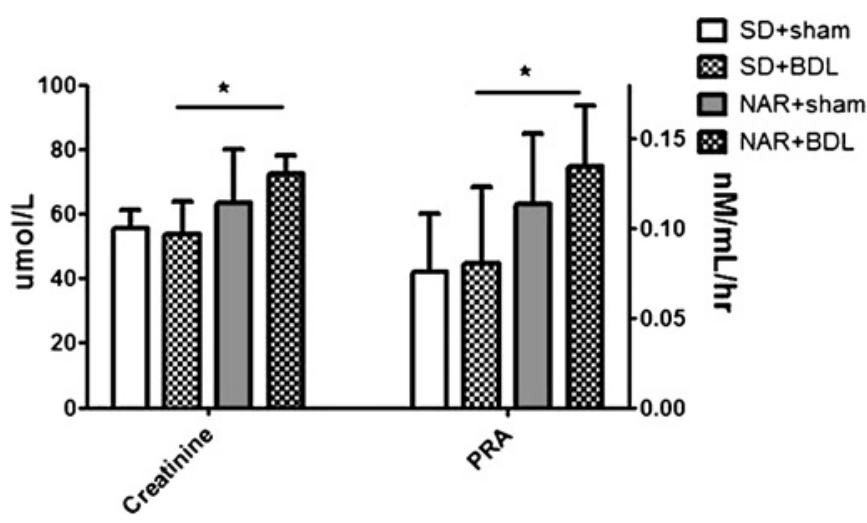

Abstract OC-068 Figure 1 Plasma creatinine concentration and plasma renin activity in the different groups of animals

Conclusion A lack of albumin was associated with a raised PRA and a marked deterioration in systemic haemodynamics and renal function after liver injury (BDL), despite normal total plasma protein concentration. This worsened outcome in the absence of albumin strongly supports the central role of albumin in 